\title{
Shift Dynamics of Capillary Self-Alignment
}

\author{
Gari Arutinov ${ }^{1,2, *}$, Massimo Mastrangeli ${ }^{3}$, Edsger C.P. Smits ${ }^{1}$, Gert van Heck ${ }^{1}$, \\ Herman F.M. Schoo ${ }^{1}$, Jaap J.M. den Toonder ${ }^{2}$, and Andreas Dietzel ${ }^{4}$ \\ ${ }^{1}$ Holst Center/TNO, High Tech Campus 31, 5656 AE Eindhoven, The Netherlands \\ \{gari.arutinov, edsger.smits, gert.vanheck, herman.schoo\} atno.nl \\ ${ }^{2}$ Eindhoven University of Technology, Microsystems, 5600 MB Eindhoven, The Netherlands \\ $\{j \cdot m \cdot j \cdot d . t o o n d e r @ t u e . n l\}$ \\ ${ }^{3}$ BEAMS, Université Libre de Bruxelles, Av. Fr. Roosevelt 50, B1050 Bruxelles, Belgium \\ \{massimo.mastrangeli@ulb.ac.be\} \\ ${ }^{4}$ Technische Universität Braunschweig, Institut für Mikrotechnik, \\ 38124 Braunschweig, Germany \\ \{a.dietzel@tu-braunschweig.de\}
}

\begin{abstract}
This paper describes the dynamics of capillary self-alignment of components with initial shift offsets from matching receptor sites. The analysis of the full uniaxial self-alignment dynamics of foil-based mesoscopic dies from pre-alignment to final settling evidenced three distinct, sequential regimes impacting the process performance. The dependence of accuracy, alignment time and repeatability of capillary self-alignment on control parameters such as size, weight, surface energy and initial offset of assembling dies was investigated. Finally, we studied the influence of the dynamic coupling between the degenerate oscillation modes of the system on the alignment performance by means of pre-defined biaxial offsets.
\end{abstract}

Keywords: capillarity, self-alignment, dynamics, fluidics, packaging.

Surface tension-driven self-alignment (SA) is a simple, accurate and cost-effective technique for heterogeneous integration and stacking of dies onto pre-patterned substrates. A vast class of fluidic self-assembly processes was thoroughly investigated and developed since the early 90's, and many applications were successfully demonstrated [1-2]. Capillary SA rapidly emerged as a remarkable way to overcome the accuracy/throughput trade-off limiting established techniques of pick-and-place microassembly [3]. It indeed combines the manipulative dexterity of robotic handling for fast and rough component pre-alignment with the highly-accurate and passive final alignment yielded by the relaxation of a liquid droplet over a shape-matching patterned confinement site [4]. Die fetching and registration can thus be partly parallelized, leading to increased efficiency in the integration of innovative microsystems [5]. A more comprehensive knowledge of capillary SA needs further modeling and experimental testing. Within the past two decades researchers made significant progress in the former direction [6-10]. However, most of modeling works are based on quasi-static simulations, whereas the dynamics of the process has been rarely tackled so far.

\footnotetext{
* Corresponding author.
} 
In this paper we present an experimental study of the in-plane dynamics of capillary SA. We used transparent mesoscopic dies as components to be assembled onto shape-matching receptor sites patterned onto the substrates. Using water droplets in air and following the experimental procedure described in Fig. 1, mm- to cm-sized square foil dies could be aligned onto the patterned carriers with accuracy better than $30 \mu \mathrm{m}$, i.e. with high accuracy relatively to their lateral dimensions, and comparable to dicing tolerances. We analyzed the full uniaxial dynamics of capillary SA from die pre-alignment to final settling, evidencing the presence of three distinct, sequential regimes and highlighting their impact on the process performance. The dependence of accuracy, alignment time and repeatability of capillary SA on control parameters such as size, weight and surface energy of assembling dies, and their initial misalignment was additionally investigated. Finally, we studied the influence of the coupling between the degenerate in-plane oscillation modes of the dynamic system on the alignment performance by means of pre-defined biaxial offsets.

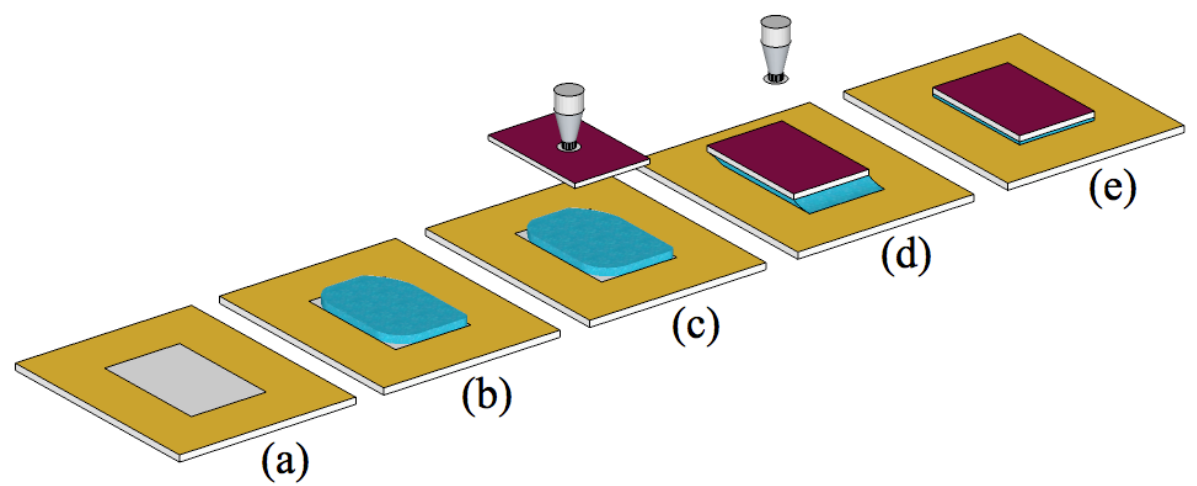

Fig. 1. Sketch of the process steps for capillary self-alignment of a foil die: (a) patterning of the carrier substrate, (b) deposition of a droplet of assembly liquid, (c) coarse alignment of a functional die, (d) the liquid wets foil die forming a meniscus, (e) self-alignment of a foil die on the corresponding binding site.

Foil dies were fabricated from transparent $125-\mu$ m-thick Polyethylene Naphthalate (PEN) sheets (Teonex ${ }^{\circledR}$ Q65FA, DuPont $\left.{ }^{\circledR}\right)$. Using a frequency-tripled Nd:YAG laser, a PEN sheet was diced into a set of square-shaped foil dies ranging in dimensions from 2 to $20 \mathrm{~mm}$ with dicing accuracies down to $30 \mu \mathrm{m}$. To quantify alignment dynamics and placement accuracy, marker structures were engraved in the center of each foil die (Fig. 2b). The laser-ablated PEN foils were aligned onto Au-patterned Si monitor wafers (Fig. 2a), allowing for reproducible and stable surface conditions in the experiments (Fig. 3). The dies were handled through a homebuilt micropositioning stage equipped with integrated vacuum tweezers (SMD-VAC-HP, Vacuum Industries Inc.). A positioning base stage (XYZ 500 TIS, Quater Research and Development) providing $10 \mu \mathrm{m}$-resolved displacements along three axes was used to pre-align the dies. High-speed camera stage and image recognition software were used to track and analyze the process dynamics. 

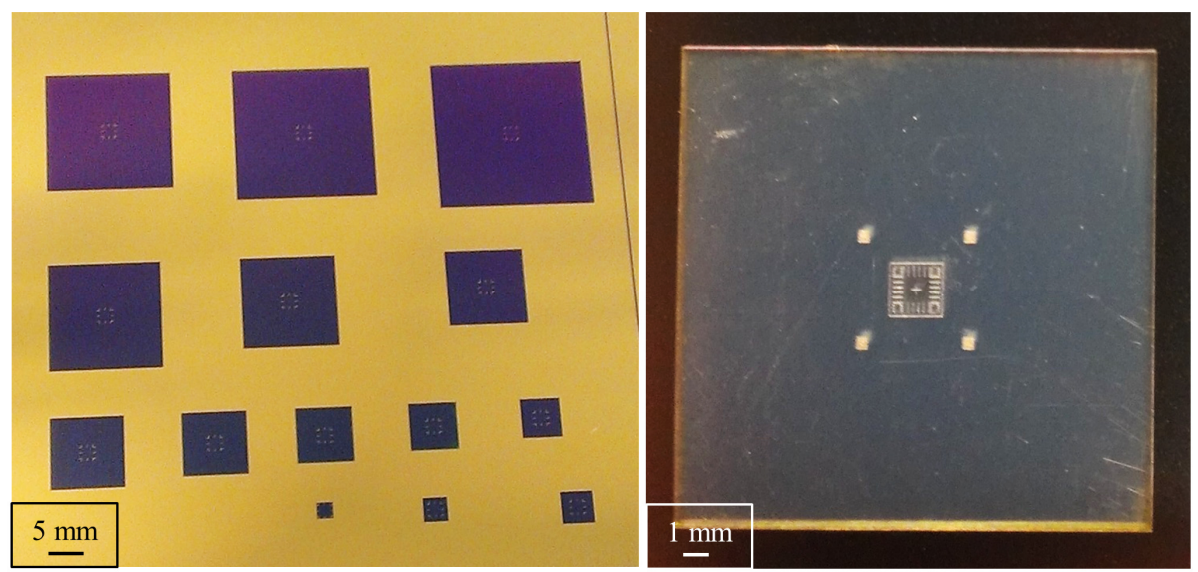

Fig. 2. (a) Optical image of a Si wafer with top patterned Au layer functionalized with perfluoro-decanethiol SAM. Dark square areas are hydrophilic $\mathrm{SiO}_{2}$ binding sites. Marker structures consisting of $50 \mu \mathrm{m}$-wide Au lines are patterned at the center of each site. (b) Optical image of a transparent, 125- $\mu$ m-thick PEN die cut by a frequency-tripled Nd:YAG (355 nm, $25 \mathrm{~ns})$ laser. The laser was also used to engrave marker structures at the center of the foil die. The combined markers allow registering the position of the foils with accuracies down to $20 \mu \mathrm{m}$.

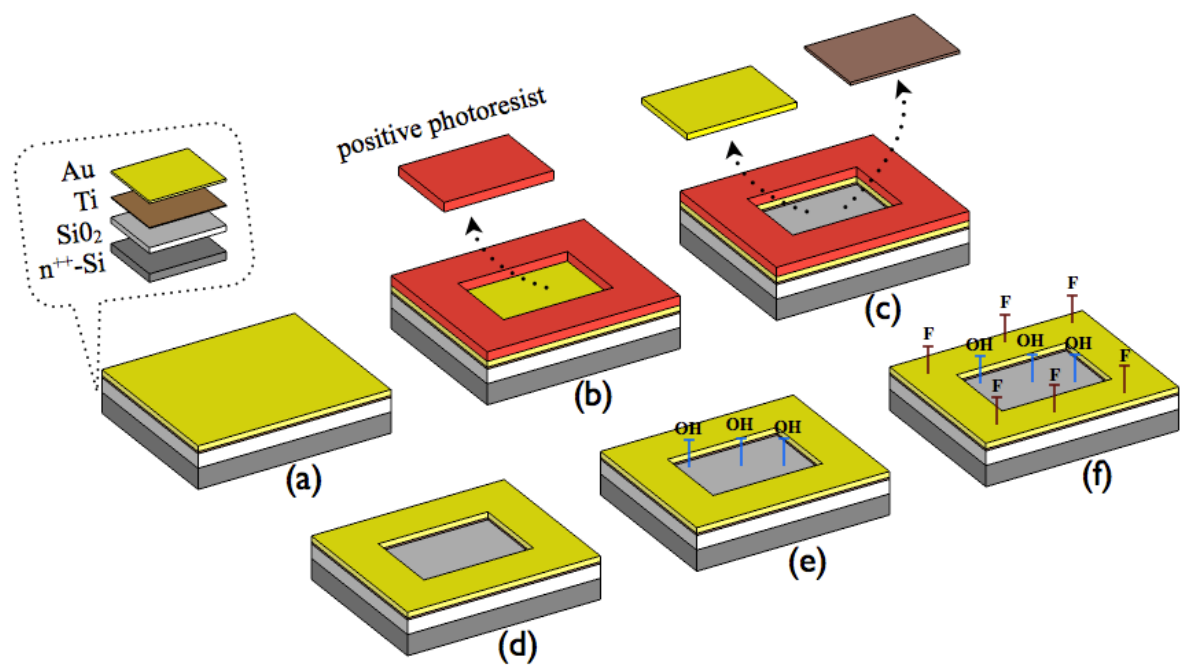

Fig. 3. Schematics of the patterning process for the Si substrate. (a) $\mathrm{Ti}(5 \mathrm{~nm})$ and $\mathrm{Au}(100 \mathrm{~nm})$ layers are sequentially sputtered over a $1 \mu \mathrm{m}$-thick layer of $\mathrm{SiO}_{2}$ thermally-grown on the $\mathrm{Si}$ wafer. (b) Positive photoresist is spin-coated, patterned and developed to define the binding sites. (c) Au and Ti are removed from the binding sites by wet etching. (d) The remaining photoresist is striped. (e) The substrate is cleaned by $\mathrm{O}_{2}$ plasma. (f) Selective deposition of a fluorinated alkanethiol self-assembled monolayer on the Au surface. Relative dimensions out of scale for illustration purposes. 
Analysis of recorded SA trajectories (Fig. 4a) revealed that, upon release of the foil die from a relatively-large uniaxial offset from the target position, the ensuing dynamics of capillary SA can be divided into three separate regimes [11]. Transient wetting comes first, whereby the foil stays initially at rest after landing on the droplet (Fig. 4a). After contact with the bottom surface of the foil die, the liquid forms a bridge and spreads across the slit geometry. The relaxation of the liquid meniscus continues till it reaches and pins over at least one of the boundaries of the foil die. This event marks the beginning of the uniaxial translational motion of the foil die. In this second regime the foil die moves with a constant acceleration (see inset in Fig 4a) and hence describes a parabolic trajectory. It is therefore subjected to a constant lateral force, which arises from the deformation of the meniscus and the gain in surface energy as surface wetting progresses. This constant acceleration regime fades as the die reaches the vicinity of the target position and dissipative viscous forces become comparable with the driving capillary forces. The final dynamic regime can be described by a damped harmonic oscillation (DHO, Fig 4a) observed in flip-chip assembly, as well [6-7]. By tailoring surface wettability, we contextually showed that transient wetting and constant acceleration regimes are strongly dependent on and can be tuned by the surface energy of the bottom surface of the assembling foil dies (Fig. 4b). Particularly, increased surface energy-i.e., higher hydrophilicity and lower contact angle-decreases the total time-to-alignment.

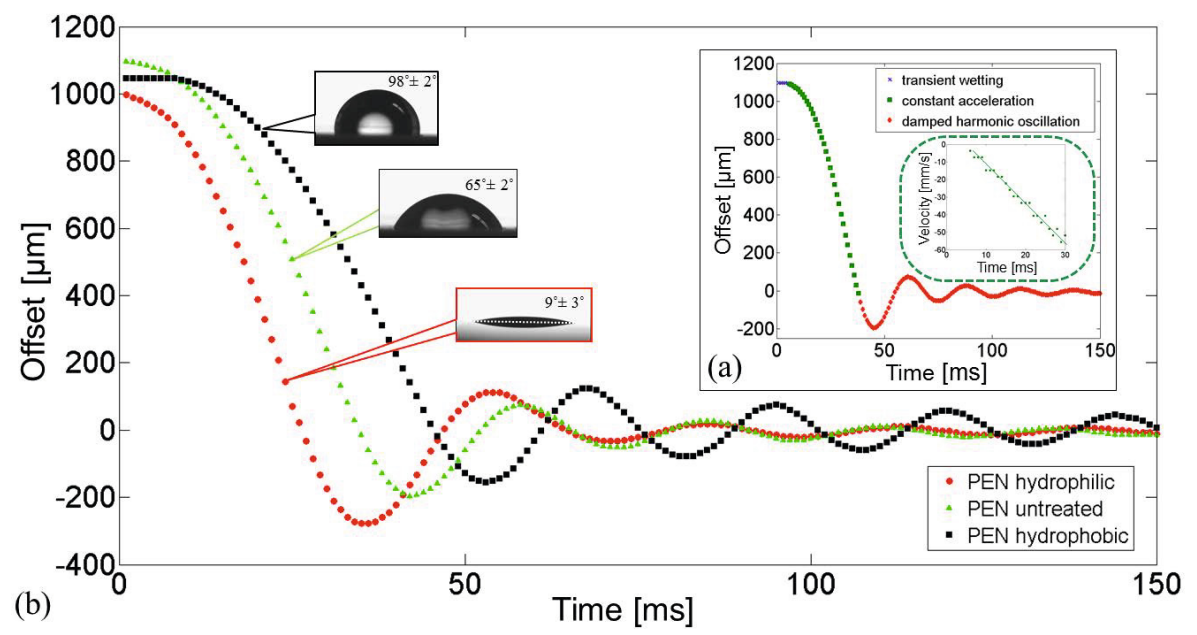

Fig. 4. (a) Self-alignment trajectory of $18 \times 18 \mathrm{~mm}^{2}$ foil die $\left(0.80 \mathrm{mg} / \mathrm{mm}^{2}\right)$ extracted from self-alignment process recorded by high-speed camera. (inset) Numerical derivative of the parabolic regime depicted in (a), evidencing linear progression of velocity in time. (b) Selfalignment trajectories of $18 \times 18 \mathrm{~mm}^{2} \mathrm{PEN}$ foil die $\left(0.80 \mathrm{mg} / \mathrm{mm}^{2}\right)$ with no coating and hydrophilic and hydrophobic coating.

In contrast to the sub-millimetric case, the dies used hereby had a Bond number $B o=\rho g L^{2} / \gamma>>1$. Hence the inertia of the considered mesoscopic foil dies was expected to significantly affect their self-alignment dynamics. Sizes and weights of foil 
dies were thus investigated to find a reliable process window. Foil dies were coarsely aligned manually with an initial offset of $20 \%$ of their lateral size, preventing offsetdependent alignment failure [12]. The water layer thickness $(125 \mu \mathrm{m})$ was the same for all binding sites. As seen in Fig. 5, the assembly yield was substantially lower for the smallest and the largest foils. Moreover, for the smallest foils the accuracy of the initial offset was hardly reproducible (standard deviation of about $30 \mu \mathrm{m}$ ). Consequently, the SA process was considered unreliable.

To investigate the influence of inertia on the DHO regime, equal-sized PEN dies with mass densities varying from 0.18 to $0.98 \mathrm{mg} / \mathrm{mm}^{2}$ were pre-aligned over corresponding binding sites with prefixed initial offset of $950 \pm 50 \mu \mathrm{m}$. Their SA trajectories evidenced a net impact of inertial effects on the oscillation period (Fig. 6).

We additionally investigated the SA dynamics for quasi-1D cases. A foil die was repeatedly released at different, pre-fixed small offsets along the secondary axis while the initial offset along the principal axis was kept constant within loading precision. The corresponding SA data revealed no divergence of the trajectories along the principal axis during the constant acceleration regime (Fig. 7a) in spite of significant differences in the dynamics along the secondary axis (Fig. 7b). Hence we derive that non-negligible yet relatively-small positioning errors along the secondary axis barely affect the lateral alignment dynamics of square foil dies along the principal axis.
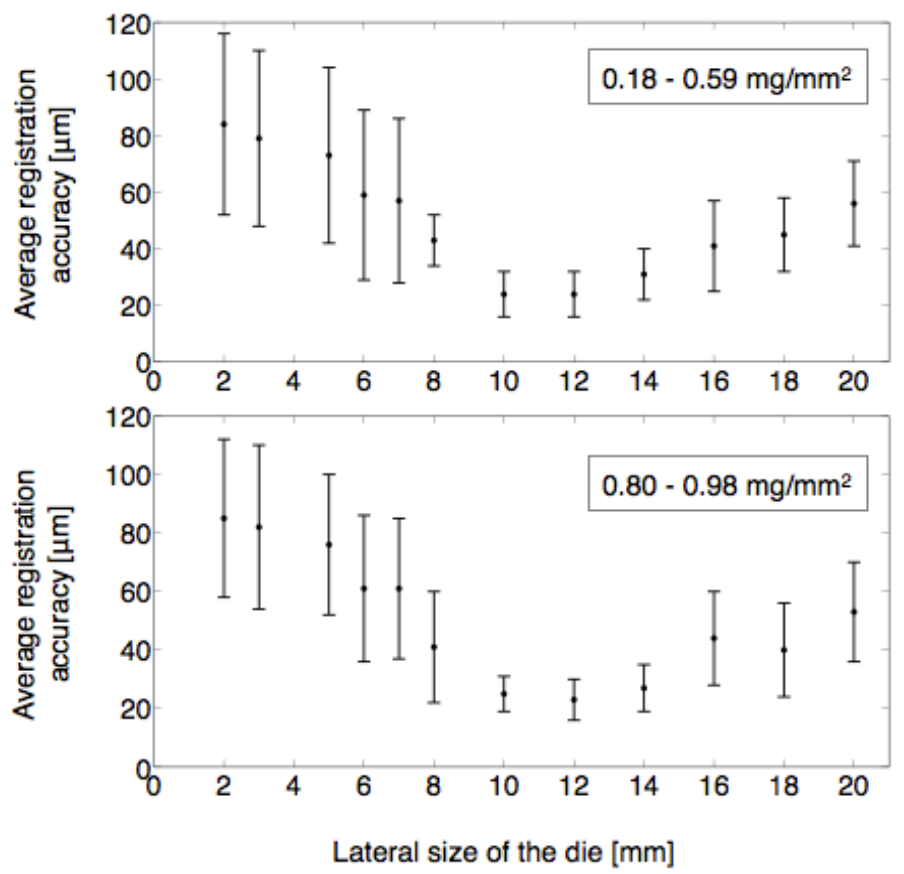

Fig. 5. Average registration accuracy for self-aligned foil dies of varying sizes and weights. Foil dies of each size and weight were self-aligned at least ten times from an initial offset of about $20 \%$ of foil die lateral size. A $125 \mu \mathrm{m}$-thick layer of water was used in all cases. 


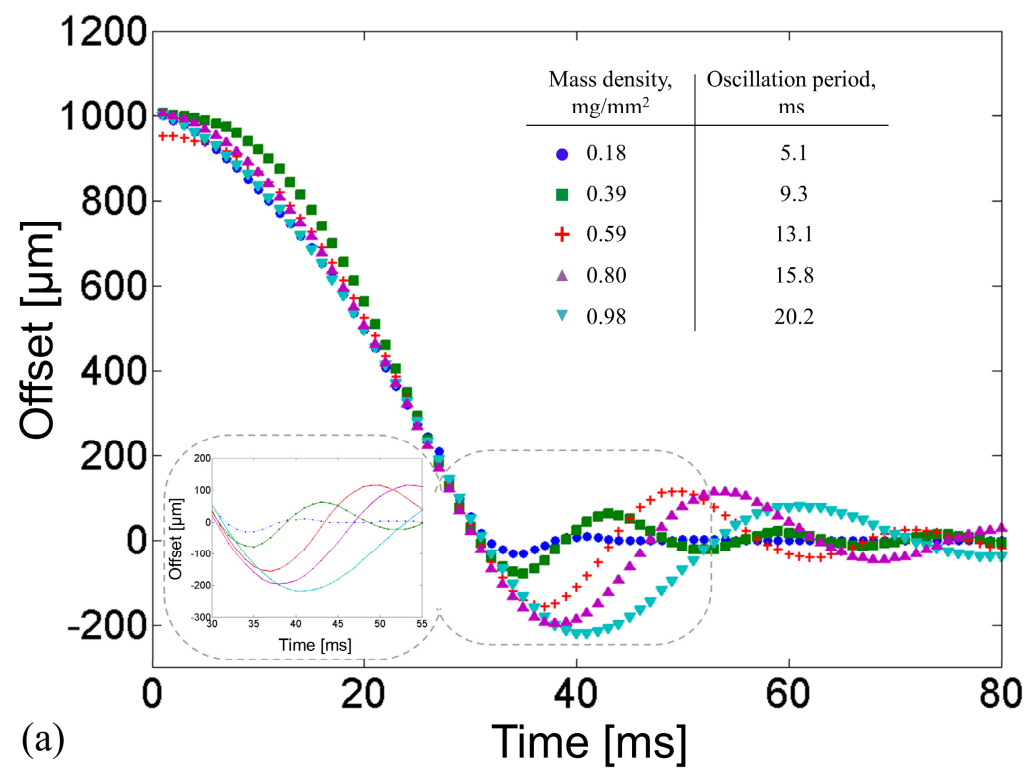

Fig. 6. Self-alignment trajectories of foil dies with different mass densities (see inset), and corresponding oscillation periods as extracted from experimental trajectories (shown in inset)

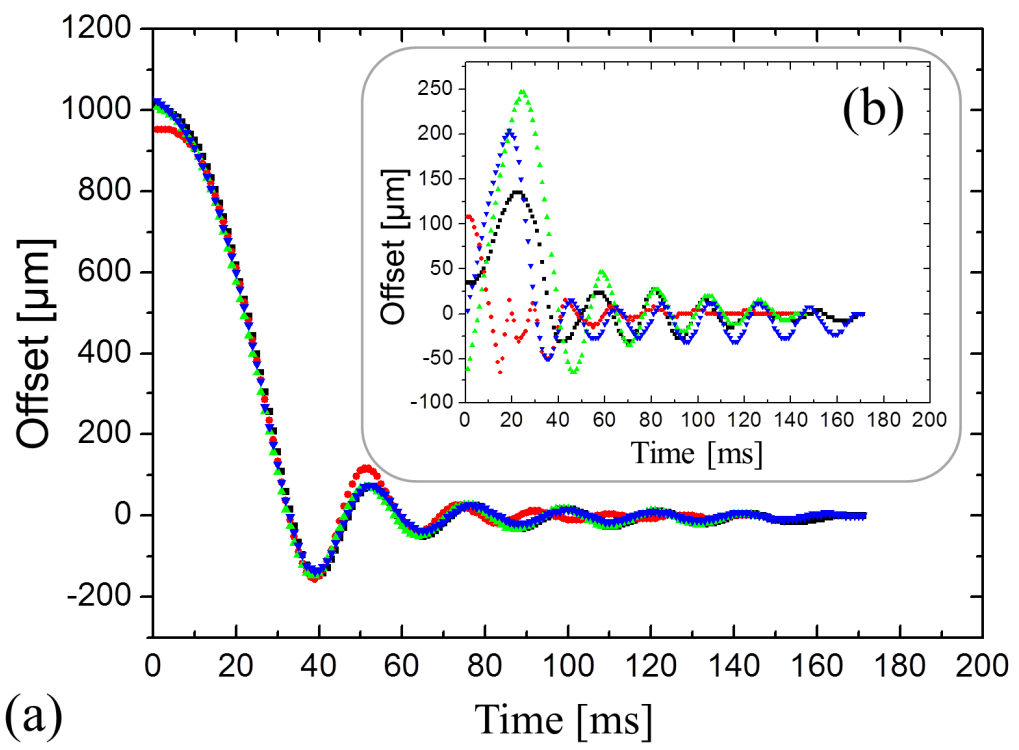

Fig. 7. Self-alignment trajectory of a foil $\left(0.80 \mathrm{mg} / \mathrm{mm}^{2}\right)$ die as recorded by high-speed camera along (a) principal and (b) secondary axis. The die was repeatedly released at various initial offsets along the secondary axis, while initial offsets along main axis was kept the same within loading precision of the micropositioner. 
As a generalization of the previous case involving weak coupling between the degenerate in-plane oscillation modes, we analyzed the SA dynamics for the case of initial die offset along the main diagonal direction (i.e. $45 \mathrm{deg}$ ) - i.e. for equal, relatively-large initial die offsets along both axes, and consequent strong coupling between the modes. Recorded SA trajectories evidence an initially linear translation of the die along the diagonal direction, followed by an inward spiraling trajectory as the die ultimately approaches the center of the target site (Fig. 8a). A DHO equation provided also in this case a very good fitting for the projection of the oscillatory SA trajectory along the main axes (Fig. 8b). Final accuracy for diagonal self-alignment matched the best uniaxial case. Notably, for a given, small radial offset from the center of the binding site, the capillary restoring force ensuing from a diagonal displacement is larger than for a uniaxial displacement. This may make diagonal alignment preferable to the uniaxial, as it may avoid issues of the latter for small offsets [12].
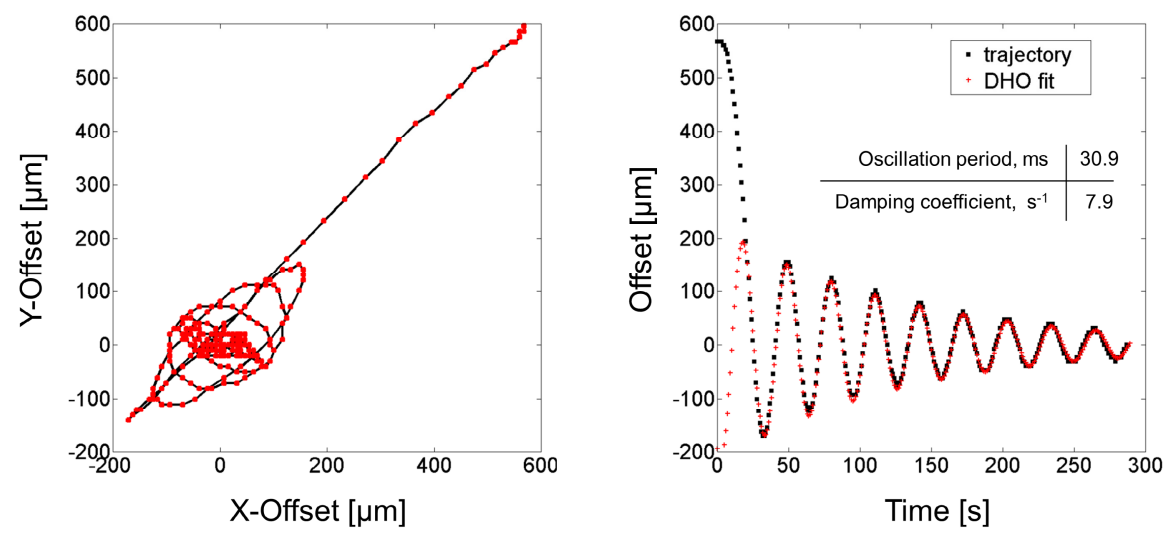

Fig. 8. (a) The phase plane of self-alignment trajectory of a foil $\left(0.80 \mathrm{mg} / \mathrm{mm}^{2}\right)$ die as recorded by high-speed camera along diagonal axis, and (b) self-alignment trajectory fitted with damped harmonic oscillation (DHO) approximation and corresponding oscillation period and damped coefficient as extracted from trajectories (shown in inset). The die was released along the diagonal axis, where initial offsets along secondary and principle axes were in principle equal.

In conclusion, our investigation of the dynamics of capillary SA significantly extends the understanding of the process available in literature, and suggests additional parameters to control its performance, such as the optimal uniaxial offset window and the surface energy of the die. Though our analysis focused on millimetric dies, most of the results can directly be applied to the case of much smaller dies-in fact, the use of larger beside transparent vehicles just makes the phenomena more apparent with respect to the microscopic scale, thus easier to detect and study. Finally, this new body of knowledge can further ease or extend the compliant adoption of the technique for industrial microsystem manufacturing. 


\section{References}

1. Mastrangeli, M., Abbasi, S., Varel, C., van Hoof, C., Celis, J.-P., Böhringe, K.F.: J. Micromech. Microeng. 19, 081001, 37 p. (2009)

2. Crane, N.B., Onen, O., Carballo, J., Ni, Q., Guldiken, R.: Microfluid. Nanofluid. 14, 383-419 (2013)

3. Morris, C., Stauth, S.A., Parviz, B.A.: IEEE Trans. Adv. Pack. 28, 600-611 (2005)

4. Sariola, V., Jääskeläinen, M., Zhou, Q.: IEEE Trans. Robot. 26, 965-977 (2010)

5. Mastrangeli, M.: Surface tension-driven self-assembly. In: Lambert, P. (ed.) Surface Tension in Microsystems, ch. 12, pp. 227-253. Springer (2013)

6. Lin, W., Patra, S.K., Lee, Y.C.: IEEE Trans. Compon. Packag. Manuf. Technol. 18, 543-551 (1995)

7. van Veen, N.: J. Electron Packag. 121, 116-121 (1999)

8. Mastrangeli, M., Valsamis, J.-B., van Hoof, C., Celis, J.-P., Lambert, P.: J. Micromech. Microeng. 20, 075041 (2010)

9. Lambert, P., Mastrangeli, M., Valsamis, J.-B., Degrez, G.: Microfluid. Nanofluid. 9, 797-807 (2010)

10. Berthier, J., Mermoz, S., Brakke, K., Sanchez, L., Frétigny, C., Di Cioccio, L.: Microfluid. Nanofluid 14, 845-858 (2013)

11. Arutinov, G., Mastrangeli, M., Smits, E.C.P., Schoo, H.F.M., Brugger, J., Dietzel, A.: Appl. Phys. Lett. 102, 144101 (2013)

12. Arutinov, G., Smits, E.C.P., Mastrangeli, M., van Heck, G., van den Brand, J., Schoo, H.F.M., Dietzel, A.: J. Micromech. Microeng. 22, 115022 (2012) 